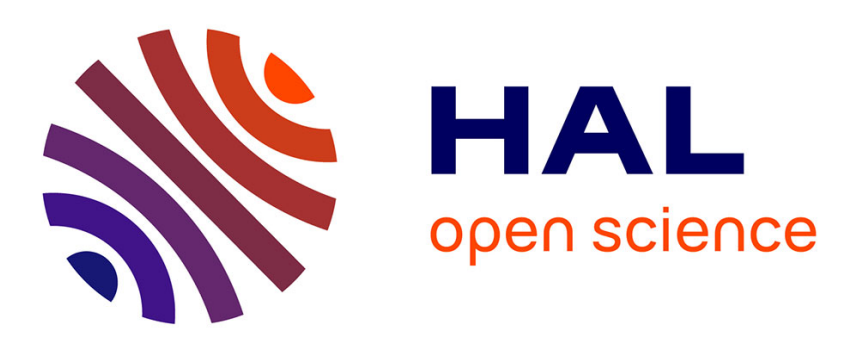

\title{
L'image du corps en neurologie: de la cénesthésie à l'image spéculaire. Apports cliniques et théoriques de la psychanalyse
}

Catherine Morin, Stéphane Thibierge

\section{- To cite this version:}

Catherine Morin, Stéphane Thibierge. L'image du corps en neurologie: de la cénesthésie à l'image spéculaire. Apports cliniques et théoriques de la psychanalyse. L'Évolution Psychiatrique, 2004, 69, pp.417-430. 10.1016/j.evopsy.2003.10.002 . hal-01519564

\section{HAL Id: hal-01519564 \\ https://hal.science/hal-01519564}

Submitted on 24 Aug 2017

HAL is a multi-disciplinary open access archive for the deposit and dissemination of scientific research documents, whether they are published or not. The documents may come from teaching and research institutions in France or abroad, or from public or private research centers.
L'archive ouverte pluridisciplinaire HAL, est destinée au dépôt et à la diffusion de documents scientifiques de niveau recherche, publiés ou non, émanant des établissements d'enseignement et de recherche français ou étrangers, des laboratoires publics ou privés. 


\title{
L'image du corps en neurologie : de la cénesthésie à l'image spéculaire. Apports cliniques et théoriques de la psychanalyse ${ }^{>}$
}

\author{
Body image in neurology: from cenesthesia to \\ specular image. Clinical and theoretical \\ contributions of psychoanalysis
}

\author{
Catherine Morin ${ }^{\mathrm{a}, *}$, Stéphane Thibierge ${ }^{\mathrm{b}}$ \\ ${ }^{a}$ Chargée de recherche, CNRS LEAPLE (UMR 8606), service de médecine physique et réadaptation, hôpital \\ de la Salpetrière, 47, boulevard de l'Hôpital, 75651 Paris cedex 13, France \\ ${ }^{\mathrm{b}}$ Chargé de recherche, CNRS LEAPLE (UMR 8606), maître de conférence des universités, UFR sciences \\ humaines et arts, université de Poitiers, 97, avenue du Recteur-Pineau, 86022 Poitiers, France
}

Reçu le 16 septembre 2003 ; accepté le 17 octobre 2003

Disponible sur internet le 18 août 2004

\section{Résumé}

Le concept de schéma corporel est actuellement bien séparé en neurologie de celui d'image du corps, chacun renvoyant à des représentations du corps distinctes. Ces représentations permettent, respectivement, à chacun de nous d'ajuster automatiquement les mouvements de son corps à son espace et d'avoir de son corps dans l'espace une représentation langagière. Le terme d'image du corps reste également employé en psychanalyse, mais avec une signification tout à fait différente. Pour la psychanalyse, l'image du corps est un des registres de l'identité, et le rôle primordial du regard de l'Autre dans l'acquisition de cette identité apparaît dans le terme d'image spéculaire. Après avoir repris l'histoire et les antécédents de ces différents termes, nous envisageons les implications en neurologie de la notion d'image spéculaire. En effet, les pathologies neurologiques de la motricité et du schéma corporel ne sont pas sans conséquences sur l'image spéculaire, ces conséquences pouvant

\footnotetext{
> Toute référence àcet article doit porter mention: L'image du corps en neurologie: de lacénesthésie à l'image spéculaire. Apports cliniques et théoriquesdelapsychanalyse.Évol psychiatr. 2004;69.

* Auteur correspondant : Mme Catherine Morin.

Adresse e-mail : catherine.morin@ chups.jussieu.fr (C. Morin).
}

0014-3855/\$ - see front matter @ 2004 Elsevier SAS. Tous droits réservés. doi:10.1016/j.evopsy.2003.10.002 
aller jusqu'à une véritable déconstruction de cette image. Une telle lecture permet de reconnaître une cohérence et une logique à certains symptômes pseudopsychiatriques des lésions hémisphériques droites.

(C) 2004 Elsevier SAS. Tous droits réservés.

\begin{abstract}
In neurology, the terms body schema and body image currently refer to two different body representations. These representations are meant to allow humans individuals to automatically adjust their bodies to space in sensorimotor activities and also have a semantic knowledge of their bodies and their body-space relationships. The term body image is also used in psychoanalysis, in a quite different maner. From the psychoanalytical point of view, body image is one of the registers of identity and the Other's regard plays a primordial role in the subject's acquisition of this identity. The crucial role of this gaze appears in the term specular image. After reminding the reader of history and the predecessors of these terms, we consider the implications of the concept of specular image in neurology. We show that neurological pathology may have effects upon specular image, up to and including a disorganization of the structure of specular image when brain lesions affect body schema. This perspective permits to find out a coherence and a logic in some psychiatric-like symptoms of right hemispheric brain lesions.
\end{abstract}

(c) 2004 Elsevier SAS. Tous droits réservés.

Mots-clés : Image du corps ; Image spéculaire ; Neurologie ; Psychanalyse ; Anosognosie

Keywords: Body image; Specular Image; Neurology; Psychoanalysis; Anosognosia

Image et corps sont des vocables du langage courant qui paraissent renvoyer chacun à un sens repérable : qui prétendrait en effet ne pas savoir du tout ce qu'est une image ou ce qu'est un corps ? Pourtant l'association des deux termes dans l'expression image du corps, qui appartient à la fois au langage de la neurologie, de la psychiatrie et à celui de la psychanalyse, prend bien des significations différentes selon l'époque, la discipline ou les présupposés théoriques des divers spécialistes qui l'ont employée ou l'emploient. Aucune de ces significations spécialisées n'est directement rapportable à l'acception courante des deux termes ainsi associés. Pour autant, aucune des disciplines concernées n'a tenté de remplacer ces mots si courants par une expression plus technique. Cela indique bien, nous semble-t-il, à quel point tout un chacun est concerné par le problème de la représentation du corps. Par ailleurs, la diversité de ses acceptions, tout comme la variété des champs scientifiques où est employée l'expression image du corps, indiquent aussi combien il est difficile à l'intuition commune d'en rendre compte ou d'en préciser le sens. Nous souhaitons examiner ici brièvement l'histoire des théories qui ont voulu éclairer les rapports entre corps et subjectivité. Ce parcours nous fera rencontrer d'autres concepts tels que cénesthésie, schéma corporel, et image spéculaire. Notre propos n'est pas ici pour autant celui de l'historien : il voudrait seulement tenter de préciser les « antécédents » de ces termes et leur portée respective. Tous survivent en effet aujourd'hui avec des sortes de domaines réservés : au traitement de la douleur la cénesthésie [1], à la neurologie l'image et le schéma corporels [2,3], à la psychanalyse l'image spéculaire, mais aussi, dans une autre acception 
que celle de la neurologie, l'image du corps ${ }^{1}$ [5]. Par ailleurs, l'expression image du corps se rencontre, le plus souvent pour faire référence à l'apparence physique, dans la littérature actuelle sur les troubles du comportement alimentaire [6] ou le handicap [7]. Pour notre part, nous souhaitons montrer ici que la notion d'image spéculaire, de par ce qu'elle permet d'élaborer en clinique et en théorie [5,8], a d'importantes implications en neurologie. Nous les examinerons à partir d'une pratique en médecine de réadaptation, touchant les suites d'accidents vasculaires cérébraux (AVC) invalidants. En effet, cette pathologie affecte non seulement le fonctionnement sensorimomoteur, mais aussi la représentation psychique du corps et de l'identité, selon des formules associatives variées.

Depuis Schilder [9], la différenciation entre schéma corporel et image du corps est un thème récurrent en neurologie, en psychologie et en psychanalyse. Chacun s'accorde depuis Bonnier [10] et Head [11] sur une hypothèse que l'on peut résumer comme suit : le cerveau, et plus particulèrement l'hémisphère droit, dispose d'une représentation nonconsciente du corps, le schéma corporel, représentation qui permet un ajustement automatique de nos mouvements à notre environnement spatial (pour ref. cf. [3]). En revanche, les concepts désignés sous le terme d'image du corps ont beaucoup varié. En neurologie, ce terme renvoie actuellement à des représentations verbales multiples du corps, de ses différentes parties et de ses rapports spatiaux à celui d'autrui, représentations mises en défaut par certaines lésions hémisphériques gauches dans l'autotopoagnosie [2]. C'est à la persistance de cette image verbale du corps que Gallagher et Cole [12] attribuent les possibilités de compensation de désafférentations sensitives massives qui rendent inopérant le schéma corporel. Bien que ces auteurs reprennent après Dolto ([13], p. 7) la distinction entre schéma « anonyme » et image «personnelle », ni schéma ni image ne font, dans leurs acceptions actuelles, de vraie place à la subjectivité, c'est-à-dire au fait que l'action comme la représentation du corps engage nécessairement le désir du sujet (cf. par exemple aussi [14]). C'est en reprenant l'histoire des théories modernes des rapports entre corps et subjectivité, que l'on trouve chez certains auteurs, sous différentes formes et en particulier sous le terme d'image du corps, la référence à une fonction spécifique de la représentation du corps dans l'économie subjective.

\section{Psychiatrie et neurologie : de la cénesthésie au schéma corporel}

\section{Cénesthésie}

On trouve dans un travail de Jean Starobinski [15] la description de l'invention et de la promotion de la cénesthésie par Hübner et Schiff (pour ref. cf. [15]) et du passage de la notion de cénesthésie à celle d'image du corps. À sa première apparition en 1794, le mot coenesthesis est ainsi défini par Hübner : « une sensibilité générale (Gemeingefühl), qui représente à l'âme l'état de son corps alors que la sensibilité la renseigne sur le monde externe et que le sens interne (inner Sinn) donne représentations, jugements, idées et concepts ». En 1873, Schiff explique comment se forme la cénesthésie :

\footnotetext{
${ }^{1}$ Lacan J. Le stade du miroir comme formateur de la fonction du Je telle qu'elle nous est révélée dans
} l'expérience psychanalytique (1949). In : ([4] p. 93-100). 
«Si par exemple, l'irradiation (de l'excitation vers les centres) se dirige vers un centre sensoriel, elle y éveillera l'image d'une couleur d'un son, d'un objet ; une impression auditive peut ainsi produire une sensation visuelle ou une impression auditive ou les deux en même temps ; une telle sensation secondaire en produira à son tour une tertiaire et ainsi de suite. De cette façon une seule sensation peut éveiller une chaîne infinie de sensations centrales d'images sensorielles et comme toute notre pensée se meut dans de telles images ou plus exactement n'est pas autre chose qu'une série d'images centrales, c'est-à-dire d'excitation de la terminaison centrale des nerfs sensitifs, il s'ensuit qu'une sensation peut produire une série de pensées qui, réunies aux sensations primitives, doivent compléter ou plutôt constituer la cénesthésie ».

C'est dans la filiation de cette conception que Séglas ${ }^{2}$ attribue une responsabilité primordiale aux troubles cénesthésiques dans la mélancolie : «par suite des troubles qui surviennent dans le domaine des fonctions organiques au début de la mélancolie », écrit-il, « l'état cénesthésique normal, de bien-être, produit par le consensus harmonique des sensations organiques fait place, une fois l'équilibre rompu, à un nouvel état cénesthésique pénible de malaise général » et cet état est « une première cause de la douleur morale ». De même, Dupré et Camus [17] décrivent les cénestopathies, maladies de la cénesthésie:

« Nous proposons de désigner sous le nom de cénestopathies les altérations de la sensibilité commune ou interne, c'est-à-dire les troubles de ces sensations qui incessamment arrivent au cerveau de tous les points du corps et qui à l'état normal ne s'imposent à notre attention par aucun caractère particulier soit dans leur intensité soit dans leur modalité. On sait de quelle importance est le domaine de la cénesthésie qui constitue au-dessous des champs de perception de la conscience le fondement primitif de notre personnalité ».

La vie psychique est ici vue comme un système d'interactions entre le corps et le monde, tous deux sources de stimulations dont la combinaison constitue le psychisme.

\section{Schéma corporel}

C'est Pierre Bonnier [10] qui ramène la représentation du corps vers celle d'une forme. Il se demande de quelle fonction normale le vertige est le trouble, et nomme cette fonction sens des attitudes. C'est dans ce cheminement qu'il introduit la notion de schéma.

«Le sens des attitudes », écrit-il, « nous fournit la notion du lieu de chaque partie de nous-mêmes et forme la base de toute orientation, tant objective que subjective et psychique. Il a pour objet la figuration topographique (rvge $\mu$ a) de notre moi. J'ai également proposé ce terme de schématie pour le genre d'images fournies par ce sens. Le mot cénesthésie ne peut avoir de signification valable en physiologie et en psychologie car il ne comporte pas la notion de figuration topographique indispensable à toute définition de corporalité ».

\footnotetext{
${ }^{2}$ Séglas J. Mélancolies sans délire (1895). In : [16] p. 282-295.
} 
Notons également que Bonnier lie explicitement la représentation du corps et ce qu'il appelle le moi :

« une chose n'acquiert d'existence réelle pour nous que par l'identité des localisations de ses divers aspects sensoriels ; la distribution topographique des choses de notre milieu les unes par rapport aux autres et par rapport à nous, qui permet l'extériorisation sensorielle, crée la notion d'objectivité ; de même la notion de subjectivité dépend de la localisation des choses en nous, et ces deux termes du moi et du non moi sont sortis des opérations les plus directes du sens des attitudes ».

Head [11] considère, pour sa part, les données posturales comme la base de la représentation du corps, et il le fait dans les termes suivants : il existe un «standard postural » auquel sont confrontées toutes les perceptions nouvelles. Cette conception, dans son esprit, est proche de la «schématie » de Bonnier. À partir de cette notion de schéma, promue séparément par Head et par Bonnier, c'est la forme qui a gagné. C'est un tournant crucial : cela veut dire que s'interpose entre le sujet et son corps une représentation inconsciente mais organisée de ce corps.

\section{Psychologie, neurologie, psychanalyse et image du corps : chemins croisés}

On peut dire qu'au cours des années 1930, et dans la suite du fil que nous venons d'évoquer, la psychologie avec Wallon [18], la psychanalyse avec Schilder [9] et Lacan ${ }^{3}$, et la neurologie avec Lhermitte [19] vont se croiser sans se rencontrer.

\section{La notion du corps propre}

En 1931, Wallon montre «Comment se développe chez l'enfant la notion du corps propre » [18]. Le corps est d'abord traité par l'enfant comme s'il était fait de parties distinctes, animées chacune d'une vie personnelle : tel enfant peut ainsi offrir des morceaux de gâteaux à ses orteils. Entre six mois et deux ans, l'enfant découvre son image dans le miroir et, à la différence du jeune chimpanzé, il s'y intéresse de façon prolongée, même après en avoir constaté le caractère fictif : il jubile devant son image. Il se retourne vers l'adulte, quêtant son assentiment devant cette image qui est la sienne. On notera ici que l'enfant s'identifie à la forme de son corps alors même que son schéma corporel au sens de Head [11] n'est pas constitué, qu'il n'a pas la notion de la droite et de la gauche [20], et qu'il ne saurait bien sûr énumérer les diverses parties du corps. Notons aussi que, si certains auteurs insistent actuellement sur le possible caractère inné d'une telle image [21,22], tous continuent à admettre que la représentation de notre corps se construit d'une façon ou d'une autre [23].

\section{L’image du corps}

En 1935, Schilder [9], neurologue et psychanalyste, introduit le terme d'image du corps et non de schéma corporel. Il insiste sur l'existence d'une image optique et non pas

\footnotetext{
${ }^{3}$ Lacan J. Le stade du miroir comme formateur de la fonction du Je telle qu'elle nous est révélée dans
} l'expérience psychanalytique (1949). In : ([4] p. 93-100). 
posturale du corps, image à laquelle la perception est rapportée. Plus généralement pour lui, «l'image du corps humain c'est l'image de notre propre corps que nous formons dans notre esprit, autrement dit la façon dont notre corps nous apparaît à nous-mêmes ». De cette image, il donne une caractérisation négative et réticente qui résume cependant très bienle problème : « image du corps » est selon lui un « terme bien fait pour montrer qu'il y a ici autre chose que sensation pure et simple, et autre chose qu'imagination : un apparaître à soi-même du corps, terme qui indique aussi que bien que passant par les sens ce n'est pas là pure perception et bien que contenant des images mentales et des représentations ce n'est pas là pure représentation ». Comme on le sait, Schilder divise son grand ouvrage en trois parties. Ces trois parties correspondent aux trois ordres de faits dont il sut apercevoir qu'ils devaient tous trois être pris en compte pour un abord correct de l'image du corps. Ces trois ordres de faits sont par lui spécifiés comme les fondements physiologiques, la structure libidinale, et la sociologie de l'image du corps. Dans les fondements physiologiques de l'image du corps, nous retrouvons le corps de la neurologie. La structure libidinale de l'image du corps s'organise autour des orifices du corps, c'est-à-dire des zones érogènes selon Freud [24], dont Schilder souligne la richesse d'expression symbolique ou imaginaire. On doit en déduire que, pour lui, le reste du corps n'est pas érogène, pas libidinalisé : de fait, pour Schilder, la méconnaissance de l'hémiplégie, comme le membre fantôme des amputés, sont des formations psychogènes liées au fait que la psyché ne saurait admettre le manque, il s'agit de ce qu'il appelle le « refoulement organique focalisé ». Ce refoulement organique et le refoulement névrotique, tout en étant différents, ont pour lui le même mécanisme. Bien que peu claire, cette formulation nous intéresse en ceci qu'elle pose implicitement qu'une perte qui porte sur la maîtrise ou la représentation du corps n'a aucune spécificité particulière. Schilder psychanalyste, autrement dit, ne touche pas au corps de la neurologie. On peut noter aussi que Freud, malgré sa formation neurologique et son attachement à définir le narcissisme ${ }^{4}$, ne s'est jamais véritablement intéressé au caractère totalisant de la représentation intuitive de notre corps, non plus qu'aux pathologies neurologiques de cette représentation. En 1939, Jean Lhermitte publie L'image de notre corps [19]. "Chacun de nous possède », écrit-il, « effleurant au seuil de sa conscience, une image, un schéma tridimensionnel de son corps ; c'est grâce à l'existence de cette image de notre corps qu'il nous est possible de sentir, de percevoir, enfin de développer notre action sur nous-mêmes et sur le monde qui nous entoure ». Il rappelle, à partir des travaux de Wallon [18], que la connaissance de la forme du corps n'est pas innée mais acquise. Tout en affirmant que cette image est inscrite dans des structures cérébrales, et en consacrant une grande part de son ouvrage à son altération par les lésions cérébrales, en particulier pariétales droites, Lhermitte note: « Il serait bien vain de chercher à découvrir dans le système nerveux un dispositif fixe et rigide comme soutien organique d'une image si variable, si chargée de sens et d'histoire que l'est en réalité l'image de notre corps ». Les données modernes concernant la neuroanatomie complexe du «physical self »[22] confirment ce postulat de Lhermitte. On peut retenir de cette définition qu'il s'agit d'une forme, mais que cette forme, étant « chargée de sens et d'histoire » ne saurait se réduire au modèle postural de Head [11]. En disant l'image du corps « chargée de sens et d'histoire », en décrivant les « asomatognosies généralisées » de telle façon qu'il est facile d’y

\footnotetext{
${ }^{4}$ Freud S. Pour introduire le narcissisme (1914). In : ([25]. p. 81-105).
} 
reconnaître aujourd'hui des dépersonnalisations psychotiques, Lhermitte, contrairement à Schilder [9], indique implicitement que l'image du corps dans son ensemble, et non pas seulement dans les zones érogènes, peut être considérée comme libidinalisée, c'est-à-dire non réductible à ce qui serait un simple donné perceptif.

\section{L'image spéculaire}

Identification imaginaire et identification symbolique

En 1949, dans « Le stade du miroir comme formateur de la fonction du Je telle qu'elle nous est révélée dans l'expérience psychanalytique $»^{5}$, Lacan établit de quelle façon l'image du corps peut être « chargée de sens », selon l'expression de Lhermitte. Il le fait à partir d'une réinterprétation des observations de Wallon [18], en termes de passage d'un corps morcelé (état réel) à l'identification à une image (virtuelle). Il souligne que cette phase du miroir est cruciale pour l'identification du sujet, c'est-à-dire pour l'acquisition de ce que nous appelons une identité : le sujet s'identifie alors à une image (identification imaginaire). C'est l'image d'un corps complet, debout et unitaire et Lacan ${ }^{6}$, précisant les termes de moi idéal et d'Idéal du moi chez Freud ${ }^{7}$ rapporte au moi idéal cette forme virtuelle. L'adulte, auprès de l'enfant, indique et reconnaît ce corps pour être celui de tel enfant singulièrement nommé : des mots, des éléments symboliques lui sont attachés, réalisant une identification symbolique. Ces traits symboliques qui représentent le sujet dans l'ordre du langage caractérisent pour Lacan l'Idéal du moi freudien. Ainsi l'infans devient-il un sujet humain, reconnaissant son corps comme un, de forme semblable à celui d'autrui, tout en étant cependant son propre corps nommé, et inscrit par là-même dans une filiation et une appartenance sexuelle. L'intervention de cette identification symbolique, qu'entérine en principe le nom propre, est attestée par les pathologies où elle est en défaut. C'est le cas des psychoses, en particulier dans les syndromes psychotiques de fausse reconnaissance de Capgras et de Frégoli : au lieu de se rapporter aux variétés de formes d'une image singulière, le nom y est distinct de l'image et produit une série indéfinie de réduplications $[5,8]$. C'est à la liaison complexe qui noue le corps réel, sa forme comme image, et ce qui le désigne dans l'ordre du langage, c'est-à-dire dans l'ordre symbolique, que la psychanalyse donne le nom d'image spéculaire ${ }^{8}$. De la neurologie, Lacan retient et le fait que l'image est inscrite dans le cortex cérébral, qu'il qualifie même de « miroir intraorganique $»^{9}$, et la méconnaissance impliquée dans cette reconnaissance de la forme : méconnaissance de l'immaturité réelle (motrice, neurologique notamment) de l'enfant au stade du miroir, méconnaissance normale du fonctionnement réel du corps, voire de son amputation réelle, comme dans les cas de membre fantôme [23]. Mais une autre méconnaissance caractérise cette identification imaginaire : c'est la méconnaissance de ce que l'image doit, ne serait-ce que du fait qu'elle est reconnue comme une, à ses déterminations

\footnotetext{
${ }^{5}$ Lacan J. Le stade du miroir comme formateur de la fonction du Je telle qu'elle nous est révélée dans l'expérience psychanalytique (1949). In : ([4] p. 93-100).

${ }^{6}$ Lacan J. Remarques sur le rapport de Daniel Lagache (1961). In: ([4] p. 647-84).

${ }^{7}$ Freud S. Le Moi et le ça. (1923). In : [26] p. 177-234).

${ }^{8}$ Lacan J. Remarques sur le rapport de Daniel Lagache (1961). In : ([4] p. 647-84).

${ }^{9}$ Lacan J. Le stade du miroir comme formateur de la fonction du Je telle qu'elle nous est révélée dans l'expérience psychanalytique (1949). In : ([4] p. 93-100).
} 
symboliques. C'est là une première différence importante entre l'image spéculaire et l'image du corps dans son acception neurologique actuelle, qui en fait une représentation seulement opérationnelle du corps.

Image du corps et objet

Une autre différence tout aussi importante tient au fait que le corps est libidinalisé ${ }^{10}$ :il est investi narcissiquement, autrement dit, le sujet est capté par la forme de ce corps ; il est également, et simultanément, représenté pour un autre, c'est-à-dire qu'il est nécessairement vécu — bien ou mal- comme objet de désir pour autrui. C'est cela qu'un corps représente fondamentalement, et qui par définition ne peut être entièrement réductible au symbole ou à l'image : ce que je représente pour l'autre, et pour le désir de l'autre, je ne peux en avoir la maîtrise ni la connaissance. C'est cela seul qui peut expliquer la brillance ou l'attrait d'un corps particulier — parfois son propre corps - aux yeux du sujet, alors qu'il s'agit primordialement d'une forme standard. Si cette forme ne peut se réduire à un standard, non plus qu'à un simple objet de perception, c'est qu'elle représente aussi le regard de l'Autre, en tant que le sujet interroge ce regard, interroge ce en quoi il plaît ou non à l'Autre, ce en quoi il répond ou non à la demande ou au désir de l'Autre. Cet x impalpable, c'est ce que Lacan, nomme, à la suite de Freud [24] mais de manière plus précise en structure, l'objet [27]. Les zones érogènes sont les zones corporelles où prendra effet la perte de cet objet ${ }^{11}$ : la bouche (lieu de la demande de nourriture et d'amour), l'œil (lieu du désir ou de la demande de l'Autre, en tant qu'ils passent par le regard), l'anus (lieu de la demande de l'Autre), l'oreille (lieu du désir ou de la demande de l'Autre, en tant qu'ils passent par la voix). L'objet qui correspondrait à ces désirs ou à ces demandes (objet que l'on peut par commodité se représenter sous les espèces du sein, de la voix, du regard, des fécès) est fondamentalement manquant. C'est ce manque que la psychanalyse désigne sous le nom de castration ${ }^{12}$. C'est parce que cet objet manque à l'image que celle-ci peut prendre consistance, et c'est en tant que le corps visible chez nous ou chez autrui représente ce manque qu'il peut être désirable. La représentation symbolique de ce manque est le phallus - comme pur élément symbolique, c'est-à-dire comme signifiant ${ }^{13}$. Par exemple, pour ce qui nous intéresse ici, la représentation du corps humain debout, érigé, symétrique, droit, «normal», est l'effet dans le registre imaginaire de la mise en place de ce signifiant pour un sujet. En tous cas, il est essentiel de souligner que toute autre « positivation » de l'objet perdu a des effets déstructurants qui vont de l' « inquiétante étrangeté » freudienne ${ }^{14}$ à la dépersonnalisation, en passant par toutes les variétés du sentiment d'étrangeté isolées par la psychiatrie classique (pour ref., cf. [5]). On peut donc caractériser ainsi l'apport spécifique de la psychanalyse à la question rebattue des rapports entre corps et psyché : les principales coordonnées constituant le sujet ne sont pas deux — son corps et son psychisme-, mais trois. Ces coordonnées sont : l'objet - soit ce qui pose la question de sa valeur et de son statut au regard de l'Autre-, l'image de son corps et les nominations qui le représentent

\footnotetext{
${ }^{10}$ Freud S. Pour introduire le narcissisme (1914). In : ([25]. p. 81-105).

${ }^{11}$ Lacan J. Subversion du sujet et dialectique du désir (1960). In : ([4] p. 793-827).

${ }^{12}$ ibidem

${ }^{13}$ Lacan J. La signification du phallus (1958). In : [4] p. 685-6.

${ }^{14}$ Freud S. L'inquiétante étrangeté (1919). In : [28] p. 208-63.
} 
dans l'ordre symbolique. Dans les « Remarques sur le rapport de Daniel Lagache $»{ }^{15}$, Lacan soutient que le parcours d'une psychanalyse, en amenant le sujet à s'appréhender du lieu de l'Autre, peut lui faire apercevoir un moment cette triplicité.

Nous voudrions soutenir ici que la notion d'image spéculaire permet de penser de façon économique et cohérente les divers troubles de l'identité observés soit en neurologie, soit en psychiatrie. En effet, l'un d'entre nous a pu [5], en utilisant comme grille de lecture les divers registres de l'image spéculaire, ramener les diverses formes des troubles psychiatriques de l'identification aux avatars d'une structure commune, repérable dans toute psychose $^{16}[8]$ : la désintrication de l'image et du nom, corrélative de la venue au premier plan de l'objet positivé. Utilisant la même grille pour lire les observations neurologiques classiques de troubles de l'image du corps [30], il avait suggéré que la même structure pouvait s'y retrouver [5].

\section{Implications en neurologie}

C'est cette hypothèse que nous voudrions ici mettre à l'épreuve, à partir d'observations cliniques récentes recueillies dans un service dédié à la réadaptation après hémiplégie vasculaire. En effet, les conditions de cette réadaptation permettent de recueillir et de mettre en perspective un matériel clinique pertinent pour notre propos, du fait notamment de la durée d'hospitalisation et des diverses relations transférentielles instaurées au cours de cette période.

La clinique de l'hémiplégie vasculaire nous propose deux grands modes de perturbations subjectives : le premier est un mode non spécifique, induit par l'hémiplégie en tant que handicap moteur de survenue traumatique, et la notion d'image spéculaire nous paraît heuristique pour décrire de façon précise la dépression post-AVC. Nous envisagerons en particulier les modalités du deuil dans cette pathologie. Le second mode, qui se situe hors de la problématique du deuil, est celui d'une véritable déconstruction de l'image spéculaire, spécifique de certaines localisations lésionnelles particulières, qui laisse apparaître crûment les rapports normalement masqués entre image du corps et objet. Cette seconde clinique nous permettra d'envisager les apports de la psychanalyse à la physiopathologie de la méconnaissance apparente de l'hémiplégie décrite par Babinski [31] sous le nom d'anosognosie.

Le handicap hémiplégique : un désaccord entre les aspects imaginaire et symbolique de l'image spéculaire

Tout handicap moteur altère la représentation que le sujet peut se faire de son identité et de son corps, en bousculant la maîtrise, la prestance, bref la valeur phallique de l'image spéculaire. Ceci désaccorde les aspects imaginaires et symboliques de cette image et de l'identité. Le récit autobiographique d'une femme clouée au lit dans son enfance par un mal de Pott [32], illustre de façon saisissante un tel désarrimage entre l'image corporelle et les

\footnotetext{
${ }^{15}$ Lacan J. Remarques sur le rapport de Daniel Lagache (1961). In: ([4] p. 647-84).

${ }^{16}$ Czermak M. Signification psychanalytique du syndrome de Cotard. In : [29] p. 205-36.
} 
signifiants attachés à l'idéal du moi : «Quand je me levai enfin et que j’eus réappris à marcher, un jour je pris en main une glace et j'allai vers un miroir en pied pour me regarder. (...) Tout se passa sans bruit sans cris, je n'ai pas hurlé de rage quand je me suis vue, je me suis senti abattue, c'est tout. Cette personne dans le miroir, ce ne pouvait pas être moi. Intérieurement, je me sentais quelqu'un d'ordinaire, en bonne santé, veinarde — pas du tout comme celle du miroir, oh ! Non. Pourtant chaque fois que je me tournais vers le miroir, c'étaient mes propres yeux qui me renvoyaient mon regard brûlant de honte ». De tels bouleversements sont habituellement rangés sous l'étiquette de la dépression, du retentissement affectif du handicap, du deuil, termes dont la valeur descriptive n'est pas ici en discussion. Toutefois, il est légitime de s'interroger sur les rapports précis des symptômes psychologiques avec l'image corporelle, dans la mesure où le corps lui-même est altéré dans ces pathologies. Les travaux de l'une d'entre nous, fondés sur une épreuve d'autoportrait [33], montrent que l'image du corps est bel et bien altérée par toute hémiplégie. Deux points sont à souligner concernant les altérations observées : d'une part, malgré l'asymétrie du handicap hémiplégique, ce sont les deux mains, les deux pieds ou la bouche qui peuvent être omis ; d'autre part, les autoportraits sont verticaux, que leurs auteurs soient ou non capables de se tenir debout. La conservation de la valeur phallique (symétrie et verticalité) de l'image corporelle montre bien en l'occurrence la force de la méconnaissance attachée à l'identification imaginaire : le réel, la particularité de ce qui est perdu n'apparaît pas, du moins à ce stade, sur le portrait. De plus, ces altérations ne sont pas spécifiques : elles peuvent se voir non seulement dans d'autres handicaps aux conséquences corporelles différentes (paraplégie) mais aussi chez des sujets normaux [34] et dans des circonstances telles que le vieillissement ou la dépression [35]. Ceci montre que le corps, dans sa valeur représentative, intervient dans la symbolisation et l'imaginarisation de tout deuil ou de toute dépression. Surtout, ce type d'autoportrait nous semble illustrer de façon ramassée les caractéristiques de la dépression normale des patients hémiplégiques dans les suites immédiates de l'AVC : le manque des deux mains s'y observe tout comme chez les sujets normaux, chez qui les liens de ces lacunes de la représentation avec la castration sont audibles dans le discours [34]. C'est-à-dire que le sujet, comme dans tout deuil, est confronté à une nouvelle version du deuil fondateur de la perte de l'objet. Si la représentation en image fait apparaître les aspects non spécifiques du handicap hémiplégique, le discours des patients sur leur maladie fait, lui, apparaître des traits spécifiques à l'hémiplégie. En étudiant le maniement des sujets grammaticaux et des verbes, l'une de nous a constaté [36] que les verbes connotant l'état, la maladie et la souffrance étaient préférentiellement associés à des sujets indéterminés : " Je suis surtout gênée parce qu'on est tributaire de tous les siens », par exemple. Cette formulation, typique de l'hémiplégie, diffère de celle des blessés médullaires, où c'est l'action et non l'état qui semble faire problème (exemple : «J'ai ma main droite qui fonctionne très mal, je l'utilise quand même pour me nettoyer la figure, mais malheureusement y a des choses qu'on peut pas faire soi-même »).

Ceci nous semble témoigner d'un désaccord entre les registres imaginaire et symbolique concernant l'image du corps : celui qui parle ne se reconnaît pas dans son image altérée par le handicap. Cette analyse se démarque à la fois de celles qui réduisent les difficultés de ces patients à une altération de l'humeur d'origine lésionnelle, cérébrale [37] et de l'interprétation purement psychologique en termes de dépression réactionnelle. Elle a des consé- 
quences très importantes en médecine de rééducation, puisqu'elle déplace le souci thérapeutique de la question du traitement de la dépression à celle du dialogue au jour le jour, dans le cadre des échanges thérapeutiques, avec des patients dont l'identité est mise en cause par la maladie.

L’hémiplégie gauche et le syndrome hémisphérique droit : un déliement de l'image et de l'objet

Certaines lésions hémisphériques droites causent, en plus de l'hémiplégie gauche, des troubles spécifiques de la représentation du corps [38] : asomatognosie (méconnaissance de l'hémicorps paralysé), anosognosie (méconnaissance apparente de l'hémiplégie gauche), héminégligence (troubles de l'attention à l'hémi-espace gauche). Ces troubles déficitaires du schéma corporel s'accompagnent parfois de symptômes productifs : assimilation des membres paralysés à un objet inanimé, surnuméraire ou encombrant, ou encore personnification des membres paralysés. L'étrangeté de ces manifestations frappe neurologues et psychiatres, mais leur pathogénie semble défier la compréhension. Pour certains, ces discours sont des rationalisations [39] qui répondraient au déficit traumatique du schéma corporel. Mais, ces productions, dans la mesure même où elles nous introduisent dans le domaine de l'étrange, sont à l'opposé de ce qu'on met ordinairement sous le terme de rationalisation. Pour d'autres, ce sont des productions «totalement illogiques »[40]. Pourtant, elles s'organisent selon des structures et autour de thèmes récurrents d'un patient à l'autre, avec, dans leur apparente diversité, une cohérence interne. Nous avons ainsi décrit plusieurs cas dominés par la présence chez les patients de préoccupations orales. C'est ainsi par exemple qu'un patient présentant une négligence très invalidante de l'hémi-espace gauche décrivait sa pathologie en termes de troubles de l'oralité [41]. L'une d'entre nous [42] a également rapporté les cas de deux patients qui ne reconnaissaient pas leur main gauche paralysée pour leur et chez qui cette main était nantie de qualités orales : l'un de ces patients d'une part personnifiait son bras gauche («il a plus envie de travailler, il a pas tort il a trop travaillé aussi, il voudrait s'arrêter, il est comme moi »), et d'autre part déclara un jour qu'ayant cru voir passer « un bras gauche », il avait eu « envie de le mordre ». L'autre, pourtant droitier, gratifia son interlocutrice d'un baisemain «parce que je ne peux pas serrer la main » et, avec la même justification dessina des lèvres (« un baiser ») à côté de son autoportrait. Par ailleurs, chez plusieurs patientes asomatognosiques et anosognosiques, nous avons observé la personnification du bras paralysé sous la forme d'une enfant [43]. L'une de ces patientes, comme on lui demandait des nouvelles de ses enfants, secoua son bras paralysé en s'écriant : "Mimi, dis bonjour ». Cette patiente avait été un temps persuadée que son bras gauche était celui de sa fille Mimi, « resté collé après un câlin », et dans le même temps elle ne reconnaissait pas la véritable Mimi, disant « ce n'est pas ma fille, ça ! ». Une autre s'inventait un scénario, qui, disait-elle, la consolait, où son bras gauche était une fille née le jour de l'AVC, reposant dans le berceau de l'accoudoir du fauteuil roulant. Elle lui avait donné un nom : «feuille», puisque, disait-elle en souriant, les feuilles reverdissent chaque printemps. On remarque dans ces cas, d'une part un morcellement de l'image du corps (des lèvres à côté du visage), d'autre part des traits évoquant un objet mort ou non-animé (fille-feuille), des parties du corps autonomisées (un bras qui passe, un bras « collé ») ou animées d'une vie personnelle. Tous ces traits font partie de 
ceux qui pour Freud ${ }^{17}$ suscitent l'impression d' « inquiétante étrangeté », et pour Lacan [27] caractérisent la venue au premier plan de l'objet normalement refoulé. On constate ainsi la concomitance de l'atteinte du schéma corporel et d'une atteinte de l'image du corps, avec l'intrusion corrélative dans la réalité psychique du sujet d'un objet qui devrait normalement manquer, être refoulé, et pouvoir à ce titre susciter le désir.

Cette clinique, à la différence de celle du handicap hémiplégique, n'est pas une clinique du deuil. Le cas de notre patiente « consolée » par la présence de sa main-fille sans avoir été vraiment déprimée est exemplaire. Ceci diffère fondamentalement du processus du deuil où le sujet est pour un temps, précisément, inconsolable. Une formule d'Abraham et Torok [44] nous paraît pouvoir être appliquée à ces cas. Pour ces auteurs, le deuil normal est une introjection : introjecter, c'est pour eux « avaler la perte »; le deuil pathologique est une incorporation : incorporer c'est « imaginer d'avaler ce qui est perdu ». «C'est pour ne pas avaler la perte » écrivent-ils, « qu'on imagine d'avaler, d'avoir avalé ce qui est perdu sous la forme d'un objet ». Cette formule ramassée a le mérite de bien souligner l'antinomie entre deuil normal et conservation de l'objet. Dans le cas des bras-fille de nos patientes, l'incorporation de l'objet signe bien l'absence d'introjection au sens précité, l'absence de symbolisation de la perte, c'est-à-dire l'absence de deuil.

Le problème de l'anosognosie peut lui aussi être éclairé à partir des rapports entre objet et image. Nul ne peut discuter l'étroite corrélation avec certaines localisations lésionnelles de cette méconnaissance apparente de l'hémiplégie ou de ses conséquences. En même temps, certains restent sensibles à la dimension subjective de ce trouble $[45,46]$. KaplanSolms et Solms [46] y voient un processus de refoulement. Cette interprétation en termes de névrose nous semble en contradiction avec leurs propres observations et certaines de leurs considérations sur le syndrome hémisphérique droit en tant qu'il comporte pour eux une défaillance du processus de deuil et une régression à une « libido narcissique ». Weinstein [45] fait de l'anosognosie l'apanage de sujets à la structure psychique « rigide», incapables d'accepter l'échec. D'une façon moins psychologisante, et sans faire d'hypothèse sur la structure préexistante des patients, nous pourrions dire que l'anosognosie n'est peut-être pas sans rapport avec ce qu'on pourrait appeler un « trop d'objet ». On aura par exemple noté dans le discours d'une de nos patientes [43] le lien de l'intrusion de l'objet dans le psychisme avec la méconnaissance de la paralysie : nommer la main « feuille » (cf. supra) implique que la paralysie, tout comme la mort des feuilles en automne, n'est pas définitive. Plus généralement, on peut considérer que percevoir la réalité, c'est reconnaître des structures organisées autour de l'absence de l'objet. Ceci est vrai aussi bien des activités perceptives que cognitives [8]. Tenir compte d'une paralysie, avoir une représentation réaliste de ses conséquences suppose dès lors de pouvoir la rapporter à l'image virtuelle d'un corps symboliquement manquant. Cette image, on l'a vu, reste accessible aux patients hémiplégiques en l'absence de troubles de l'image du corps. En revanche, il est logique que la présence intrusive de l'objet interdise à certains patients de porter un regard réaliste sur leur situation. C'est ici une illustration de la distinction et de l'incompatibilité entre reconnaissance de la réalité et identification de l'objet [8].

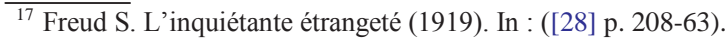




\section{Conclusion}

En un temps où se développe la recherche pour localiser les «bases neurales de la conscience de soi », ou en analyser le fonctionnement cognitif [47,48], il n'est peut-être pas inutile de rappeler que ce souci s'inscrit dans un questionnement fort ancien sur la vie psychique et le corps, sans qu'aucune de ces disciplines ait pu résoudre les apories qu'il comporte. Cet échec ne tient pas, à notre avis, à l'insuffisance qualitative ou quantitative des recherches, mais à l'hétérogénéité des registres que les neurosciences prétendent abusivement unifier. En effet, le fonctionnement du cerveau dépend de la structure et de la physiologie des neurones qu'il contient, alors que le fonctionnement psychique, lui, dépend de structures langagières, symboliques, extérieures aux cerveaux individuels. Même si ces structures ont des effets matériels sur les sujets, et donc des inscriptions cérébrales, et même si des lésions cérébrales peuvent perturber le psychisme, il n'en reste pas moins que cerveau et psychisme fonctionnent dans des registres différents. Les sciences du cerveau n'ont ainsi aucune vocation particulière à éclairer les mécanismes fondamentaux de la représentation de soi. Quant à la neuropsychologie cognitive, la représentation des activités mentales qu'elle utilise fait l'impasse sur le désir qui organise ces activités chez le sujet parlant. La psychanalyse montre au contraire comment toute représentation de soi suppose la référence au désir de l'Autre, c'est-à-dire à un lieu à la fois intime et extérieur au sujet. La clinique des troubles neurologiques de l'image du corps, comme celle des psychoses et mieux que celle de la névrose, nous révèle justement, nous pensons l'avoir montré, la présence de l'Autre au lieu même où nous sommes intuitivement le plus persuadés d'être des individus autonomes, à savoir notre corps.

\section{Références}

[1] Biasi G, Badii F, Magaldi M, Moltoni L, Marcolongo R. Un nuovo approccio al trattamento delle sindrome fibromialgica. Minerva Med 1999;90:39-43.

[2] Buxbaum LJ, Coslett HB. Specialised structural descriptions for human body parts: evidence from autotopoagnosia. Cogn Neuropsychol 2001;18:298-306.

[3] Coslett HB. Evidence for a disturbance of the body schema in neglect. Brain Cogn 1998;37:527-44.

[4] Lacan J. Ecrits. Paris: Seuil, coll. « Le champ freudien »; 1966.

[5] Thibierge S. Pathologie de l'image du corps. Étude des troubles de la reconnaissance et de la nomination en psychopathologie. Paris: PUF, coll. « Psychopathologie »; 1999.

[6] Stein D, Orbach I, Shani-Sela M, Har-Even D, Yarulasky A, Roth D, et al. Suicidal tendencies and body image and experience in anorexia nervosa and suicidal female adolescent inpatients. Psychother Psychosom 2003;72:16-25.

[7] Keppel C, Crowe F. Changes to body image and self-esteem following stroke in young adults. Neuropsychol Rehab 2000;10:15-31.

[8] Thibierge S. L'image et le double. La fonction spéculaire en pathologie. Toulouse: Erès, coll. « Psychanalyse et clinique »; 1999.

[9] Schilder P. The image and appearance of the human body. Studies in the constructive energies of the psyché. London: Kegan Paul; 1935.

[10] Bonnier P. Le sens des attitudes. Nouvelle Iconographie de La Salpêtrière 1902;15:146-83.

[11] Head H, Holmes G. Sensory disturbances from cerebral lesions. Brain 1911;34:102-254.

[12] Gallagher S, Cole J. Body image and body schema in a deafferented subject. J of Mind and Behav 1995;16:369-90.

[13] Dolto F. L'image inconsciente du corps. Paris: Masson; 1984.

[14] Paillard J. Body schema and body image: a double dissociation in deafferented patients. In: Gantchev GN, Mori S, Massion J, editors, Motor control today and to morrow. Sofia: Academic Publishing House; 1999. p. 197-214. 
[15] Starobinski J. Le concept de cénesthésie et les idées neuropsychologiques de Moritz Schiff. Gesnerus 1977;34:2-20.

[16] Séglas J. Leçons cliniques sur les maladies mentales et nerveuses. Paris: Asselin et Houzeau; 1895.

[17] Dupré E, Camus P. Les cénestopathies. Encéphale 1907;12:616-31.

[18] Wallon H. Comment se développe chez l'enfant la notion du corps propre. J Psychol. Paris 1931;28 : 705748.

[19] Lhermitte J. L'image de notre corps. Paris: L'harmattan; 1998.

[20] Lurçat L. L'enfant et l'espace. Le rôle du corps. Paris: PUF; 1979.

[21] Gallagher S, Butterworth GE, Lew A, Cole J. Hand-mouth coordination, congenital absence of limb, and evidence for innate body schemas. Brain Cogn 1998;38:53-65.

[22] Melzack R. Phantom limbs and the concept of a neuromatrix. Trends Neurosci 1990;13:88-92.

[23] Ramachandran VS, Hirstein W. The perception of phantom limbs. The D.O. Hebb lecture. Brain 1998;121: $1603-30$.

[24] Freud S. Trois essais sur la théorie sexuelle. Paris: Gallimard, coll. « Folio essais »; 1987.

[25] Freud S. La vie sexuelle. Paris: PUF, coll. « Bibliothèque de psychanalyse »; 1985.

[26] Freud S. Essais de psychanalyse. Paris: Payot; 2001.

[27] Lacan J. L'angoisse. Séminaire inédit. Paris 1962.

[28] Freud S. L’inquiétante étrangeté et autres essais. Paris: Gallimard, coll. « Folio Essais »; 1990.

[29] Czermak M. Passions de l'objet. Éudes psychanalytiques des psychoses. Paris: éditions de l'Association Freudienne; 1986.

[30] Hécaen H, de Ajurriaguerra J. Méconnaissances et hallucinations corporelles. Intégration et désintégration de la somatognosie. Paris: Masson; 1952.

[31] Babinski J. Contribution à l'étude des troubles mentaux dans l'hémiplégie organique cérébrale (anosognosie). Rev Neurol (Paris) 1914;1:845-8.

[32] Butler Hathaway K. The little locksmith. A memoir. London: Aurum Press; 2001.

[33] Morin C, Pradat-Diehl P, Robain G, Bensalah Y,Perrigot M. Stroke hemiplegia and specular image: lessons from self-portraits. Int J Hum Aging Dev 2003;56:1-41.

[34] Morin C, Bensalah Y. Self-portrait in adulthood and aging. Int J Hum Aging Dev 1998;46:45-70.

[35] Clément JP, Marchan F, Boyon D, Monti P, Léger JM, Desrouesné C. Utilization of the Draw a Person Test in the elderly. Int Psychogeriatr 1996;8:349-64.

[36] Morin C, Salazar-Orvig A. Paroles de patients hémiplégiques : discours et position subjective. Sci Soc Sante 1996; $14: 47-78$.

[37] Robinson RH, Kubos KL, Starr LB, Rao K, Price TR. Mood disorders in stroke patients. Importance of location of lesion. Brain 1984;107:81-93.

[38] Bogousslavsky J, Clarke S. Syndromes majeurs de l'hémisphère mineur. Encycl Med Chir (Elsevier, Paris) 1998; Neurologie 17-022-E-10:7 p.

[39] Halligan PW, Marshall JC, Wade DT. Unilateral somatoparaphrenia after right hemispheric stroke: a case description. Cortex 1995;31:173-82.

[40] Laplane D. L'étrange en neurologie. Étud Psychother 1998;17:23-32.

[41] Morin C, Taillefer C, Vallat C, Helsly N, Thibierge S, Pradat-Diehl P. Qu'est-ce qu'un «gauche » ? Ann Readapt Med Phys 2001;44:192-204.

[42] Morin C, Durand E, Marchal F, Timsit S, Manai R, Pradat-Diehl P, et al. Asomatognosie et troubles de l'oralité. Une lecture psychanalytique. Ann Readapt Med Phys 2002;46:12-23.

[43] Morin C, Pradat-Diehl P, Mazevet D, Gautherin D, Weill-Choulamountry A, Dubail M, et al. Un enfant dans le bras. Un trouble féminin de l'image du corps en neurologie ? Bull Assoc Lacan Int 2003;101:13-22.

[44] Abraham N, Torok M. Introjecter-incorporer : deuil ou mélancolie. Nouv Rev Psychanal 1972;6:111-22.

[45] Prigatano GP, Weinstein EA, Edwin A. Weinstein's contribution to neuropsychological rehabilitation. Neuropsychol Rehab 1996;6:305-26.

[46] Kaplan-Solms K, Solms M. Clinical studies in neuro-psychoanalysis. Introduction to a depth neuropsychology. London: Karnac Books; 2000.

[47] Vogeley K, Fink GE. Neural correlates of the first-person perspective. Trends Cogn Sci 2003;7:38-42.

[48] Smythies J. Consciousness: some basic issues. A neurophilosophical perspective. Conscious Cogn 1999;8:164-72. 